médecine/sciences 1986; $2: 106$

\section{Une nouvelle variété d'amylose}

L'amylose généralisée est caractérisée par les dépôts dans de nombreux tissus d'une substance dite amyloïde reconnaissable à ses propriétés tinctoriales et à sa structure. Au cours des deux dernières décennies, les études biochimiques ont montré l'existence de plusieurs substances amyloïdes et ont permis d'identifier diverses protéines qui en sont les constituants principaux et qui possèdent chacune un précurseur plasmatique : la protéine $A L$ qui dérive des chaînes légères des immunoglobulines, la protéine AA qui dérive d'une protéine présente dans le plasma, et la protéine $\mathrm{AF}$ qui dérive de la préalbumine. Ces résultats biochimiques ont conduit à une nouvelle classification des amyloses. Chez certains malades hémodialysés depuis de nombreuses années, on voit se développer un syndrome du canal carpien obligeant à une cure chirurgicale; une infiltration amyloïde est souvent mise en évidence. La surcharge amyloïde peut également atteindre d'autres articulations et, dans de rares cas, être généralisée [I]. Récemment, Gejyo et coll. ont isolé la substance amyloïde infiltrant le canal carpien d'un tel malade : la protéine isolée est différente des protéines amyloïdes jusqu'alors identifiées; son poids moléculaire est d'environ I I 000 daltons; sa composition est très proche de celle de la $\beta_{2}$-microglobuline; la séquence des 16 acides aminés qui forment l'extrémité $\mathrm{N}$-terminale est totalement identique à celle de la $\beta_{2}$-microglobuline [2]. D'autre part, Connors et coll. ont montré que la $\beta_{2}$-microglobuline humaine peut après dialyse et concentration, se transformer in vitro en des fibrilles non ramifiées de 8 à $\mathrm{r} 0 \mathrm{~nm}$ de diamètre possédant les caractères tinctoriaux et ultrastructuraux de la substance amyloïde [3]. Enfin, Bardin et collaborateurs ont utilisé la technique d'immunofluorescence pour démontrer que dans six cas sur sept l'antisérum anti- $\beta_{2}$ microglobuline se fixait sur les dépôts amyloïdes synoviaux provenant de malades hémodialysés [4].

On dispose donc de preuves convaincantes que l'amylose des malades hémodialysés dérive - et est constituée en grande partie - de $\beta_{2}$-microglobuline. En cas d'insuffisance rénale, la concentration plasmatique de cette protéine est élevée; l'hémodialyse périodique à l'aide de certains dialyseurs ne permet pas de ramener ce taux à la normale. Peuton prévenir l'amylose des hémodialysés en assurant une meilleure épuration de la $\beta_{2}$-microglobuline grâce à l'emploi de membranes de dialyse plus perméables?

$$
\text { J.-P. G. }
$$

I. Hervé JP, Clèdes J, Bourbigot B, et al. Systemic amyloidosis in the course of maintenance haemodialysis. Nephron 1985; $40: 494-5$.

2. Gejyo F, Yamada T, Odani S, et al. A new form of amyloid protein associated with chronic haemodialysis was identified as $\beta_{2}$-microglobulin. Biochem Biophys Res Commun 1985; 129 : jo1-6. 3. Connors LH, Shirahama $T$, Skinner $M$, Fenves A, Cohen AS. In vitro formation of amyloid fibrils from intact $\beta_{2}$-microglobulin. Biochem Biophys Res Commun 1985; 131 : 1063-8.

4. Bardin T, Küntz D, Zingraff J, et al. Synovial amyloidosis and $\beta_{2}$-microglobulin in long-term haemodialysis patients. Arthritis Rheum (sous presse).

\section{Albinisme et voies optiques}

Les malades atteints d'albinisme ont un bon champ visuel mais une vision binoculaire défectueuse. Chez le sujet normal une grande partie des axones provenant des cellules ganglionnaires de la rétine temporale passent $d u$ même côté du cerveau que l'œil d'origine; il en résulte une vision controlatérale. Chez l'albinos la plupart de ces fibres gagnent le côté $d u$ cerveau opposé à l'ail d'origine, d'où une proportion trop élevée de vision ipsilatérale. On admet qu'il existe une relation, peut-être même de causalité, entre le défaut de pigmentation rétinienne et l'anomalie anatomique. S'il en est ainsi, les hétérozygotes de l'albinisme normalement pigmentés ne doivent pas présenter cette anomalie On connaît de nombreux modèles animaux d'albinisme, notamment chez la souris; leurs voies optiques sont elles aussi déviées. Pour une telle étude, on a pris des chats, dont plusieurs variétés (en particulier les Siamois) ont la mutation albinos. A Salt Lake City, Leventhal et coll. ont utilisé des chats dépourvus, à l'état homozygote, de toute activité de tyrosinase, dont la rétine est donc complètement dépigmentée, mais qui ont à l'état hétérozygote une pigmentation apparemment normale. Par des méthodes biochimiques et électrophysiologiques ils ont pu détecter et même rendre visible la localisation rétinienne des fibres en relation avec les zones visuelles du cerveau, noyau géniculé dorsolatéral dans le thalamus, et cortex visuel. Chez les chats hétérozygotes, ils ont constaté qu'un nombre anormal de cellules répondent dans la rétine temporale controlatérale; il en résulte un certain degré de vision ipsilatérale. Ces résultats font penser que la modification de projection des fibres ne provient pas directement du défaut de pigmentation mais d'un effet, encore inconnu, du gène de l'albinisme ou d'un autre gène qui lui serait étroitement lié.

Dans quelle mesure ces études expérimentales peuvent-elles s'appliquer à l'homme? On a décrit au moins six types différents d'albinisme oculocutané. Les deux principaux sont les types tyrosinase - avec complète absence de pigmentation (type IA) et tyrosinase + où la pigmentation est diminuée mais non absente (type II). Les observations sur le chat visent le type tyr-, dont la fréquence à l'état hétérozygote est évaluée à $I \%$ dans la population humaine. Celle du type II est environ deux fois plus élevée. Une proportion de I à $3 \%$ de la population pourrait donc être soumise à des troubles modérés de la vision binoculaire en relation avec un gène de l'albinisme à l'état hétérozygote. Sur un plan plus général, ces travaux tendent à suggérer que des individus hétérozygotes pour une maladie apparemment récessive peuvent présenter des anomalies du système nerveux central, et cette notion ne se limite certainement pas à l'albinisme.

J.-C. D.

Leventhal AG, Vitek DJ, Creel DJ. Abnormal visual pathways in normally pigmented cats that are heterozygous for albinism. Science 1985; 229 : 1395-7. 